\title{
1-(2) Dopamine Metabolism in Brain of Parkinsonism with Special Reference to Vitamin $B_{6}$ and L-DOPA-treatment
}

\author{
Fumio Kuzuya, Tamotsu Wakita and Hirohisa Kawahara \\ The Third Department of Internal Medicine, Nagoya University \\ School of Medicine, Showa-ku, Nagoya, Japan
}

There are many reports concerning the effects of L-DOPA on Parkinson syndrome, and L-DOPA is considered as one of the most effective drugs in the treatment of Parkinson syndrome.

However, the undesirable effects of large doses of L-DOPA upon human bodies should be considered.

Since L-DOPA belongs to amino acid family, if a large dose of L-DOPA is administered to human bodies, this will result in the destruction of amino acid balance in vivo and inhibition of transaminase and decarboxylase which are key enzymes in amino acid metabolism. Moreover, it is reported that $90 \%$ of L-DOPA, which was administered orally, was metabolized to dopamine in intestinal cells and only $10 \%$ was absorbed and utilized as L-DOPA. Therefore, the amount of L-DOPA which enters into brain is very small comparing to the amount of administration.

Then, the authors investigated the metabolic speed and affinity to organs of L-DOPA.

${ }^{14} \mathrm{C}$-L-DOPA in a dose of $50 \mu \mathrm{Ci} / 100 \mathrm{~g}$ body weight was administered to rats intraperitoneally. After 30 minutes, autoradiography was observed.

The uptake of ${ }^{14} \mathrm{C}$-L-DOPA to kidney medulla was most significant, and on the contrary, the uptake of liver and brain was very small.

In pyridoxine deficient rats, the uptake was greater than in control rats.

It is supposed that the vessel wall through which L-DOPA is transported, has the activity of L-DOPA-decarboxylase.

The authors determined L-DOPA-decarboxylase activity in human aorta and rat brain.

The activity in human aorta was as same as that in rat brain.

From these facts, it is supposed that only 10\% of L-DOPA which was administered orally, is absorbed and, further, trapped by vessel wall and some \% may be inactivated by decarboxylation in vessel walls, therefore the amount of L-DOPA which can reach brain will be very small.

Next, the authors investigated the metabolism of vitamin $B_{6}$ and catecholamine when L-DOPA was administered to the patient suffering from Parkinson syndrome.

Tryptophan load test in the patients administered with L-DOPA showed the positive results, namely, $\mathrm{B}_{6}$ deficient state. After the subcutaneous injection of 
pyridoxal phosphate $30 \mathrm{mg} / \mathrm{day}$, the clinical signs of Parkinson syndrome became worse, and dopamine excretion in urine decreased remarkably in spite of that very large amount of dopamine was excreted in urine before the injection of pyridoxal phosphate.

In order to elucidate the mechanism of these clinical observations, the uptake of L-PODA into brain and the activity of dopamine synthesis was investigated in the pyridoxine deficient rats. The specific activity of ${ }^{14} \mathrm{C}-\mathrm{L}$-DOPA in the pyridoxine deficient rats was higher than in the control animals. Moreover, the synthesis activity of dopamine was also higher in the pyridoxine deficient rats.

These results were also confirmed by the fluorometric determination of catecholamine. Since L-DOPA-decarboxylase is vitamin $\mathrm{B}_{6}$ enzyme, as already known, it is supposed that the activity of the enzymes may be decreased in the pyridoxine deficient rats. So it seems difficult to explain why the concentration of dopamine in brain increases in the pyridoxine deficient animals.

Then the activity of L-DOPA decarboxylase was measured by the method of Hornykiowictz.

There was no difference in the activity between pyridoxine deficient and control rats.

According to the report of Suwa in our laboratory concerning the changes of vitamin $B_{6}$ concentration in several organs during pyridoxine deficient period, the concentration of vitamin $B_{6}$ in brain and heart does not decrease but remains unchanged for very long period.

From this fact, it is supposed that the decarboxylase activity in brain also remains unchanged during pyridoxine deficient period.

Moreover, since L-DOPA decarboxylase is not a rate limiting factor in the metabolism of catecholamine, so the synthesis of dopamine enough to be effective in Parkinson syndrome will be possible in spite of pyridoxine deficient state, so long as the transportation of L-DOPA into brain is sufficient.

The authors would like to assume that the limiting factor of dopamine synthesis in brain is rather the transportation of L-DOPA into brain than the activity of LDOPA decarboxylase.

From these facts and the results of the autoradiography above mentioned, it is concluded that pyridoxine deficient state is essential factor for L-DOPA to be effective in Parkinson syndrome.

The authors would like to emphasize that in the treatment of Parkinson syndrome with the large dose and long period administration of L-DOPA, we should pay attention to the pyridoxine deficient state, and the dosage of L-DOPA and the period of L-DOPA treatment should be further investigated. 